\title{
Multiauditing Based Cloud Storage Using Dynamic Hash Table
}

\author{
Veena Mudhol R, Chandrakala B M
}

\begin{abstract}
Cloud vault is one of the standard supplication of distributed computing framework, which offers on-request offloading administrations for the two people and establishments. in spite of the fact that, utilizers don't have full confidence on the cloud specialist co-ops (CSPs) inside that it is difficult to choose either the CSPs lives up to their licit desires for information security or not. Thus, it is evaluative to think of gainful reviewing methods to help proprietors' trust and dependence in distributed storage. In this paper, we are introducing plan of evaluating for guaranteed distributed storage dependent on 2-dimensional information structure called dynamic hash table (DHT), used to record the information data for open inspecting. This plan emigrates the endorsed data from the CSP to the TPA, and along these lines amazing decrease in the computational expense and correspondence overhead. Furthermore, The deduplication innovation is used to bring down the limit and data transfer capacity essentials of the utilities by expelling tedious data and reserves just a unique imitation of them. we upgrade our structure empowers security safeguarding by homomorphic authenticator developed on the open key and atten bunch evaluating by total BLS signature strategy. Trial results demonstrate that our instrument accomplishes secure deduplication and improvement in label age.
\end{abstract}

KEYWORDS- Public auditing, Cloud security, Data storage. Deduplication of data.

\section{INTRODUCTION}

Storage depositary in cloud is an influential field of cloud computing [1], whose intent is to supply on demand data out-sourcing facility for end-users through distinctly virtualized infrastructures [1], [2]. Because of the outrageous performance and reasonable cost and of cloud depository, a increasing number of organizations and users are frequently outsource their data storage to cloud services

\section{Manuscript received April 29, 2020}

Veena Mudhol R, M.Tech. Student, Department of Information Science and Engineering, Dayananda sagar college of engineering, Bangalore, India (e-mail: veenarmudhol@gamil.com).

Chandrakala B M, Assistant professor, Department of Information Science and Engineering, Dayananda sagar college of engineering, Bangalore, India
Providers (CSP), however, as a cloud storage technology still encounters numerous challenges in security [3]. portion of the consternation is how to check whether a cloud container system and its contributor reach the constitutional expectations of users for security of data [4]. The major issues in data security include data privacy, data protection, data attainable, data placement, and secure data dissemination, hazard, data depletion, service disruption, unauthorized attacks, and the data malversation issues. Hence from user's point of view security, honesty, privacy and confidentiality of the preserved data on the cloud must be considered essential requirements. To procure all of the above requirements, latest methods or techniques should be developed and to be accomplished. Data auditing technique is initiated in Cloud computing that acts with the solid data storage. Auditing is a operation of inspecting the user data which is agreed by the data owner or a TPA. It assists to keep integrity of stored data on the cloud. The TPA is an entity which can act in favour of the client or owner of data, who has all the required expertise knowledge, capabilities and professional mastery that are required to handle the functions of integrity verification, which reduces the burden of the client. It must be crucial that TPA should and frequently systematically audit the data in the cloud on request of user. A. Motivation Distributed cache service is one of the significant facilities provided by the distributed computing, where the customers can easily arrange themselves as the clump and share the data among themselves. Now a days, as many customers are sharing the data, cloud storage utility is associated by expanding capacity of information cached at distant servers. Hence, one critical challenge of today's distributed depository utility is to manage the ever-evolving capacity of data. Instead of maintaining many information duplicates with the similar content, deduplication deletes monotonous records by maintaining only one physical replica and indicating the other repetitious documents to that copy. This paper focuses on efficient auditing and deduplication on the information uploaded by information proprietor as well as checks for deduplication of the existing customers' blocks. B. Contribution In this paper, we suggest Secure Deduplication and Auditing of data Shared in Cloud mechanism that supports secure document level and block level deduplication. Our contributions are compiled as follows: (i)We present a public auditing scheme, which can thoroughly assists functions like dynamic auditing of data, batch auditing and data deduplication. (ii) We design DHT a data structure to track data premises for auditing in the 
TPA to achieve efficient data updating and instant auditing (iii) We propose Secure Deduplication and Auditing of Shared Data in Cloud (STLDAS) scheme that supports secure document level and block level deduplication. (iv) The algorithm supports secure deduplication and has reduced appreciably the time cost of tag generation. Experimental analysis manifests the adeptness and efficacy of Deduplication and Auditing of Shared Data in Cloud mechanism. C. Organisation The list of the paper is arranged as follows: We explain the Related works in Section 2 that provides the pros and cons on existing integrity auditing and deduplication schemes. In Section 3, we discuss the earlier models and their drawbacks. In Section 4, we discuss several preliminaries. In Section 5 we explain, Problem statement and System model that illustrates the functioning of the architecture and provides the specifics about the design goals. In Section 6, we explain scheme details of our Secure Two Level Deduplication and Auditing of Data in Cloud. In Section 7, we explain the Security analysis. In Section 8, we list out the results of experimental evaluation. Conclusions are given in Section 9.Highlight a section that you want to designate with a certain style, and then select the appropriate name on the style menu. The style will adjust your fonts and line spacing.

\section{RELATED WORK}

Submit your manuscript As our work is joined with both dynamic Auditing and deduplication, we contemplate the works in two of these regions in the accompanying areas. Confirmable data proprietorship and Proofs of Retrievability (PoR) were initially recommended by Ateniese et al., [5] and Juels et al., [6]. In their strategies, the homomorphic verification technique was joined to limit both the transmission and retribution cost. In this way, various options of PDP and PoR techniques are built to build the proficiency and overhaul the presentation of central methodologies, for example, allowing open approval [7] and supporting data update [8]. Jian Shen et al.,[9] proposed plot which contains open evaluating with bunch inspecting Heartbeat square less confirmation, where information elements are effectively bolstered. The epic unique structure incorporates an area exhibit, and doubly connected information table with this system, computational and correspondence overheads can be significantly limited. Examination of security indicates that plan can finish up the given productivity practically speaking with wanted properties. However, the understanding comprises of a two stages design or arrangement and check stage, of out of which just the confirm stage initiates cost of correspondence. Taek-youthful youn et al., [10] proposed a diagram that performs both deduplication of information and open examining of information. The plan performs challenge-reaction conventions utilizing the BLS signature-based homomorphic straight authenticator. The outsider examiner for directing open review, so as to customers. This plan fulfills all the essential security prerequisites. in any case, this plan expands the computational overhead at the distributed storage server. Deduplication in cloud and other stockpiling stages is a task where incessant or copy information is expelled from an information stream to limit the measure of physical information put away in an arrangement or framework. Notwithstanding, customer side deduplication is joined by the reveal of side channel data. Halevi et al., [11] built up the verification of ownership component that lets a shopper adequately demonstrate to a server that the specific client possesses this record. Venugopal et al., [12] use delicate figuring techniques for information mining applications. Geeta et al., [13] have performed broad survey on the most recent techniques in data reviewing and security in distributed computing. Y. Zhu et al.,[14] recommended a composition that groups the information premises for examining utilizing the IHT, and stores them in the TPA rather than the CSP. in this manner, it can limit the computational expenses and correspondence overhead. be that as it may, its refreshing activities (especially, the inclusion and cancellation ones) are inefficacious, since they would actuate the revision of mean of N/2 parts in the IHT, where $\mathrm{N}$ shows the quantity of squares, because of the liner structure of the IHT. In addition, the capacities would consequently change the grouping quantities of few squares, and inevitably makes the recalculations of their names, which would makes additional computational expenses of the CSP and pointless correspondence overhead.

\section{BACKGROUND WORK}

Hui Tian et al.,[15] proposed open inspecting plan for distributed storage lay on unique hash table (DHT), which is a 2-dimensional information structure set at a third equality examiner (TPA) to follow the information property data for dynamic reviewing. the proposed plan emigrates the approved data from the CSP to the TPA, likewise accomplish higher refreshing productivity and energizes security conservation by incorporating the homomorphic authenticator found on the open key with the arbitrary veiling made by the TPA, and perform group evaluating by playing out the total BLS signature system, in spite of the fact that the pursuit task on the DHT during the confirmation may cost additional time than the IHT. furthermore, this diagram does not bolster deduplication of information where capacity cost of information will be more.

\section{PRELIMINARIES}

\section{A .Bilinear maps}

Bilinear maps are the tool of pairing-based crypto, Let consider a cyclic groups $\mathrm{Ga}, \mathrm{Gb}$, and $\mathrm{Gt}$ be groups of the same order. A bilinear map from $\mathrm{Ga} \times \mathrm{Gb}$ to $\mathrm{Gt}$ is a function $\mathrm{e}: \mathrm{Ga} \times \mathrm{Gb} \rightarrow \mathrm{Gt}$ such that for all $\mathrm{u} \in \mathrm{Ga}, \mathrm{v} \in \mathrm{Gb}, \mathrm{y}, \mathrm{z} \in \mathrm{Z}$, $\mathrm{e}(\mathrm{u} \mathrm{y}, \mathrm{v} \mathrm{z})=\mathrm{e}(\mathrm{u}, \mathrm{v}) \mathrm{yz}$. Bilinear maps are called pairings because they relate pairs of elements from $\mathrm{Ga}$ and $\mathrm{Gb}$ with elements in Gt . Computational Diffie-Hellman (CDH) Problem: The Computational Diffie-Hellman $(\mathrm{CDH})$ problem is that, given $\mathrm{g}$, gm, gn $\in \mathrm{G}$ for unknown $\mathrm{m}$, $\mathrm{n}$ $\in \mathrm{Zp}$, to estimate gmn.

\section{B. Homomorphic Verifiable Authenticator (HVA)}

HVA is globaly utilized as a fundamental contruction 
obstruct for evaluating which enables an open examiner to confirm the uprightness of information put away in the cloud without retrieveing or downloading the real information. Regularly, advanced marks, (for example, RSA-based mark and BLS-based mark) are utilized to incite HVAs. combining SI and CGS units, such as current in amperes and magnetic field in oersteds. This often leads to confusion because equations do not balance dimensionally. If you must use mixed units, clearly state the units for each quantity in an equation.

The SI unit for magnetic field strength $H$ is $\mathrm{A} / \mathrm{m}$. However, if you wish to use units of $\mathrm{T}$, either refer to magnetic flux density $B$ or magnetic field strength symbolized as $\mu_{0} H$. Use the center dot to separate compound units, e.g., "A· $\mathrm{m}^{2}$."

\section{PROBLEM DEFINITION AND SYSTEM MODEL}

\section{A. Problem Definition}

Given the Cloud storage Model, the owner of the data outsources the document to the distributed server, group of customers distributes this document the main objectives are: - Public auditing Scheme that support dynamic data auditing - Data structure named Dynamic Hash Table(DHT) is designed to track data properties for auditing in the TPA - To perform secure document level or hunk level deduplication of data.

\section{B. System Model}

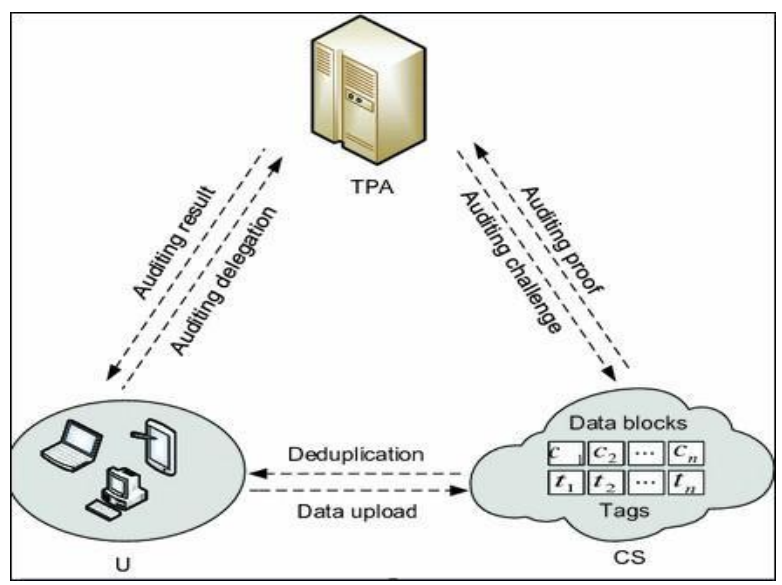

Fig.1: System Model

In this work, we focusing on the model of an beneficial public auditing scheme based on the DHT illustrated in Fig. 1, which presumes the subsequent three entities: User, who stores a considerable volume of data records in the cloud, can be an individual or a organization; Cloud Service Provider, who controles and coordinates a number of cloud servers to proffers ascendible and on-demand outsourcing data facilities for users; and Third Party Auditor, who can justify the reliability of the cloud storage utilities(CSS) tenable and devoted on behalf of the users after request. Users reatfull to the the burden of storage and computation while enjoying the storage and prolongation service by externalisation of their data into the CSP. Original customer or data owner Shared data are divided into blocks and sign with the secret key _ $\mathrm{k}$ and upload to the CSP. The CSP performs deduplication, if the file exists in its storage, the CSP intimates the original customer that the file already exist, If the file is not a duplicate then the CSP saves the file.

\section{THE ALGORITHM}

\section{A. System setup}

Consider two multiplicative groups G1, G2 of order p, and $\mathrm{e}: \mathrm{G} 1 \times \mathrm{G} 1 \rightarrow \mathrm{G} 2$ be a bilinear map. $\mathrm{H}$ is a hash function with $\mathrm{H}:(0,1) i \rightarrow \mathrm{G} 1$; assuming that document is divided into $\mathrm{n}$ blocks i,e $\mathrm{F}=(\mathrm{b} 1, \mathrm{~b} 2, \ldots . . \mathrm{bn})$ and outsourced to the CSP. Let $\mathrm{u}$ be the user or customer of the cloud.

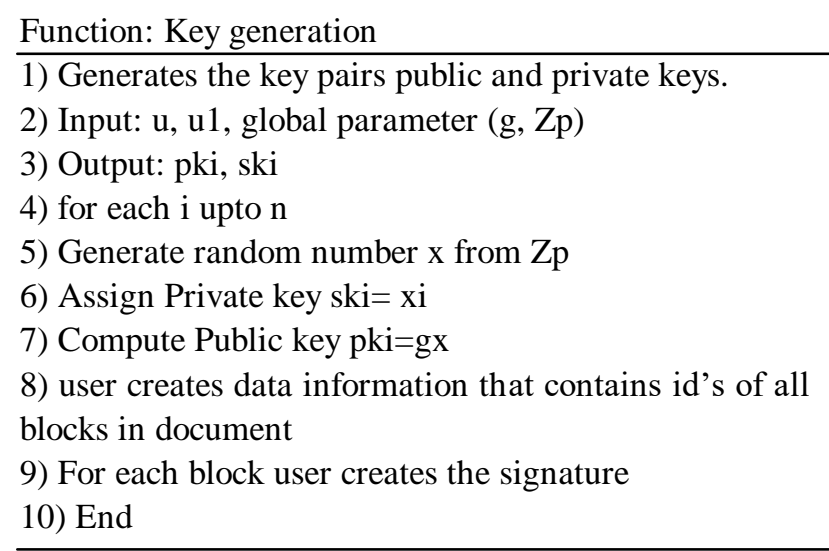

\section{B. File Uploading}

User $\mathrm{u} 1$ is considered as the information proprietor of the cluster. The information proprietor produces private key ski and public key pki for all the blocks as shown in Function Key Generation. The information proprietor executes the deduplication test by transmitting hash value of the document Hash F1 to the server (see Algorithm 1, Phase 1). If there is an identical document, the cloud user executes proof of proprietorship convention with the distributed server. If it is passed, the client is certified to retrieve this cached document without uploading the document. Otherwise, the CSP divides the file F1 into blocks, creates a tag for each block generated dynamically using Pairing Based Cryptography, where the tags are represented in the form of $\mathrm{b}(\mathrm{x}, \mathrm{y})$ where $\mathrm{b}$ is block and $(\mathrm{x}, \mathrm{y})$ is vector. The CSP verifies for the deduplication of the chunk with the respective customers. If it is the modified chunk then CSP allows to upload otherwise CSP executes the proof of ownership convention; if it is a duplicate then CSP allows the respective customers to retrieve the chunk as illustrated in Algorithm 1, Phase 2. A summary of the Notations used in the Algorithm is as shown in Table 1. 
Algorithm 1: Deduplication and dynamic Auditing.

Input: $\mathrm{F} 1=(\mathrm{m} 1, \mathrm{~m} 2, \ldots . . \mathrm{mn})$ ge, $\mathrm{mi} \in \mathrm{Zp}$, idi where $\mathrm{k} \in \mathrm{s}$ $[1, \mathrm{n}]$ Output: $\sigma \mathrm{i}$

(1) For every outsourcing document by user the following tasks are implemented:

(2) CSP examines for the deduplication of the document. If it is a current document then it moves to step 4. If the document exists then PoW convention is performed between CSP and user.

(3) After the validation that there is no duplicate copy of the document then divides the document into chunks F1 = $(\mathrm{m} 1, \mathrm{~m} 2, \ldots . . \mathrm{mn})$ and outsources to the CSP.

(4) CSP produces id and signature for every block that is created actively utilizing Pairing Based Cryptography.

(5) for each bk with idk

(6) Estimate $\sigma \mathrm{i}=(\mathrm{H}(\mathrm{idi}), \mathrm{gm} \mathrm{i}) \mathrm{x}$

(7) end for

(8)owner then outsources blocks to CSP and sends data information to TPA ,TPA stores this info in DHT.

(9) CSP validates for the deduplication of the block. If it is an update block then it moves to step 10. If the block is present then PoW convention is executed between CSP and the prevailing user.

(10) If the block does not exist in the cloud then the prevailing user uploads the modified block to cloud.

Table 1: Summary of the Notations used in the Algorithm Notation Description

\begin{tabular}{|l|l|}
\hline Notation & Description \\
\hline $\mathrm{G} 1, \mathrm{G} 2$ & Groups of order $\mathrm{p}$ \\
\hline $\mathrm{g}, \mathrm{x}$ & Generator polynomial of G1 \\
\hline $\mathrm{H}$ & $\begin{array}{l}\text { Hash function with } \mathrm{H}:(0,1)^{*} \rightarrow \\
\mathrm{G} 1\end{array}$ \\
\hline $\mathrm{PK}$ & Public key \\
\hline $\mathrm{Sk}$ & Secret key \\
\hline $\mathrm{N}$ & number of blocks in document \\
\hline $\mathrm{g}, \mathrm{x}$ & Generator polynomial of G1 \\
\hline
\end{tabular}

\section{SECURITY ANALYSIS}

We will investigating the conviction of the recommended methodology by assessing the adequacy of assault anticipation polices in this area. Hypothesis: For some rival, it is computationally absurd to establish a HVA under BLS signature procedure, if the computational DiffeHellman(CDH) supposition in bilinear gatherings holds Proof. This suggestion originates from Wang's work [6], where it show the HVA plan is experimentally exceptional, in that BLS short mark technique is secure with the assumptions that the $\mathrm{CDH}$ is a solidified issue in bilinear gatherings [17]. thus, we bar the included confirmation here. 2) Secure Deduplication: Let us assume that an adversary tries to upload his hunks of the record to the server. He sends these hunks as challenge to the CSP. After receiving these hunks, CSP runs the proof of ownership protocol and identifies that the .challenger is an attacker and informs the information proprietor. Thus, the
CSP performs deduplication securely and will protect the shared data from the adversaries efficiently.

\section{PERFORMANCE ANALYSIS}

In this segment, we present an exploratory examination of our plan. We endeavor Pairing Based Cryptography (PBC) Library [18] to perform cryptographic tasks in our show. We have utilized Intel(R) Core(TM) i3-3217U, CPU @ $1.80 \mathrm{GHz}, 2 \mathrm{~GB}$ RAM. So as to achieve 80 bit security, the prime request $p$ of the bilinear gatherings G and GT are individually picked as 160 bits long

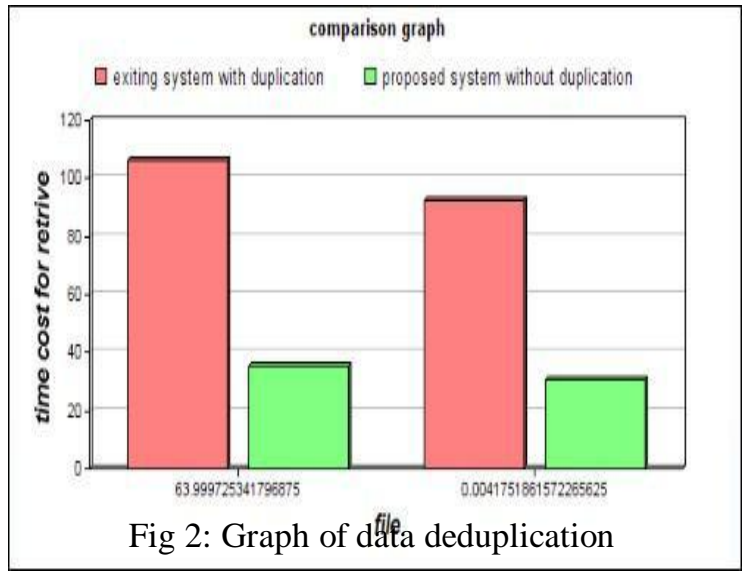

Fig. 2: Shows the experimental comparison results of data with deduplication and without deduplication stored in cloud, $\mathrm{x}$-axis represents the file size or file length, $\mathrm{y}$-axis represents time and cost.

\section{CONCLUSIONS}

With simulation to present a public auditing scheme for secure cloud storage using dynamic hash table used to the target of performing information probity. we introducing Deduplication and Data auditing in Cloud system. To list the data property information for auditing dynamically. DHT, our venture can also reach preferable performance than other schemes in the updating phase, additionally our scheme further exploits the aggregate BLS signature approach from bilinear maps to enact multiple auditing jobs simultaneously, of which the principle is to compound all the signatures on varying data blocks into a isolated one and ratify it for only one time to truncate the communication cost in the verification process.

\section{REFERENCES}

[1] H. Dewan and R. C. Hansdah, "A survey of cloud storage facilities," in Proc. 7th IEEE World Congress Serv., Jul. 2011, pp. 224-231.

[2] C. Wang, Q. Wang, K. Ren, N. Cao, and W. Lou, "Toward secure and dependable storage services in cloud computing," IEEE Trans. Serv. Comput., vol. 5, no. 2, pp. 220-232, Apr.-Jun. 2012

[3] K. Ren, C. Wang, and Q. Wang, "Security challenges for the public cloud," IEEE Internet Comput., vol. 16, no. 1, pp. 69-73, 2012.

[4] J. Ryoo, S. Rizvi, W. Aiken, and J. Kissell. "Cloud security auditing: Challenges and emerging 
approaches," IEEE Security Privacy, vol. 12, no. 6, pp. 68-74, 2014.

[5] G. Ateniese, R. Burns, R. Curtmola, J. Herring, L. Kissner, Z. Peterson, and D. Song, "Provable data possession at untrusted stores," in Proceedings of the 14th ACM Conference on Computer and Communications Security, pp. 598-609, 2007.

[6] A. Juels and B. S. Kaliski Jr, "PORs: Proofs of retrievability for large files," in Proceedings of the 14th ACM Conference

[7] G. Yang, J. Yu, W. Shen, Q. Su, Z. Fu, and R. Hao, "Enabling public auditing for shared data in cloud storage supporting identity privacy and traceability," Journal of Systems and Software, vol. 113, pp. 130-139, 2016.

[8] C. C. Erway, A. K"upc, "u, C. Papamanthou, and R. Tamassia, "Dynamic provable data possession," ACM Transactions on Information and System Security (TISSEC), vol. 17, no. 4, pp. 213-222, 2015

[9] Jian Liu, Kun Huang, Hong Rong, Huimei Wang and Ming Xian.” Privacy-Preserving Public Auditing for Regenerating-Code-Based Cloud Storage" 10.1109/TIFS.2015.2416688, IEEE Transactions on information and security 2015

[10] Taek-young youn, Ku-young chang1, Kyung-hyune rhee, and Sang uk shin " Efficient Client-Side Deduplication of Encrypted Data With Public Auditing in Cloud Storage." IEEE transactions on information forensics and security 2018.

[12] M. Sookhak, A. Akhunzada, A. Gani, M. Khurram Khan, and N. B. Anuar, "Towards dynamic remote data auditing in computational clouds," The Scientific World Journal, vol. 2014, pp. 1-12, 2014.

[13] C. M. Geeta, S. Raghavendra, R. Buyya, K. R. Venugopal, S. S. Iyengar, and L. M. Patnaik, "Data auditing and security in cloud computing: issues, challenges and future directions," International Journal of Computer (IJC), vol. 28, no. 1, pp. 8-57, 2018.

[14] Y. Zhu, G.-J. Ahn, H. Hu, S. S. Yau, H. G. An, and C.-J. Hu, "Dynamic audit services for outsourced storage in clouds," IEEE Trans. Serv. Comput., vol. 6, no. 2, pp. 227-238, Apr.-Jun. 2013.

[15 ] Hui Tian, Yuxiang Chen, Chin-Chen Chang, Hong Jiang, "Dynamic-Hash-Table Based Public Auditing for Secure Cloud Storage"IEEE transactions on services compG. O. Young, "Synthetic structure of industrial plastics (Book style with paper title and editor)," in Plastics, 2nd ed. vol. 3, J. Peters, Ed. New York: McGraw-Hill, 1964, pp. 15-64.

[16] W.-K. Chen, Linear Networks and Systems (Book style). Belmont, CA: Wadsworth, 1993, pp. 123-135.

[17] H. Poor, An Introduction to Signal Detection and Estimation. New York: Springer-Verlag, 1985, ch. 4.

[18] B. Smith, "An approach to graphs of linear forms (Unpublished work style)," unpublished.

[19] E. H. Miller, "A note on reflector arrays (Periodical style-Accepted for publication)," IEEE Trans. Antennas Propagat., to be published.
[20] J. Wang, "Fundamentals of erbium-doped fiber amplifiers arrays (Periodical style-Submitted for publication)," IEEE J. Quantum Electron., submitted for publication.

[21] C. J. Kaufman, Rocky Mountain Research Lab., Boulder, CO, private communication, May 1995.

[22] Y. Yorozu, M. Hirano, K. Oka, and Y. Tagawa, "Electron spectroscopy studies on magneto-optical media and plastic substrate interfaces(Translation Journals style)," IEEE Transl. J. Magn.Jpn., vol. 2, Aug. 1987, pp. 740-741 [Dig. 9 ${ }^{\text {th }}$ Annu. Conf. Magnetics Japan, 1982, p. 301].

[23] M. Young, The Techincal Writers Handbook. Mill Valley, CA: University Science, 1989.

[24] (Basic Book/Monograph Online Sources) J. K. Author. (year, month, day). Title (edition) [Type of medium]. Volume(issue). Available: http://www.(URL)

[25] J. Jones. (1991, May 10). Networks (2nd ed.) [Online]. Available: http://www.atm.com

[26] (Journal Online Sources style) K. Author. (year, month). Title. Journal [Type of medium]. Volume(issue), paging if given. Available: http://www.(URL)

[27] R. J. Vidmar. (1992, August). On the use of atmospheric plasmas as electromagnetic reflectors. IEEE Trans. Plasma Sci. [Online]. 21(3). pp. 876-880. Available: http://www.halcyon.com/pub/journals/21ps03-vidmar 